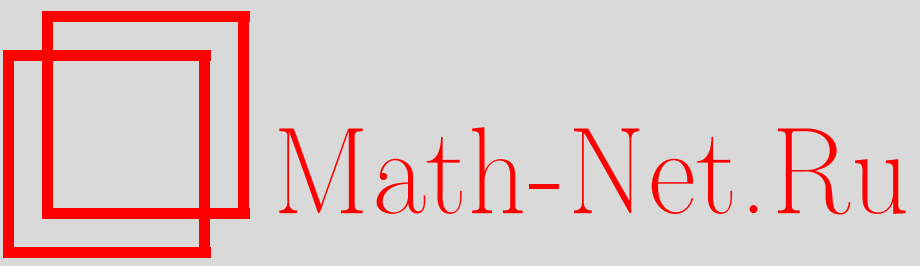

М. А. Васильев, И. В. Тютин, Лагранжево описание неприводимых массивных полей произвольного спина в размерности $2+1$, ТМФ, 1997 , том 113 , номер $1,45-$ 57

DOI: https://doi.org/10.4213/tmf1064

Использование Общероссийского математического портала Math-Net.Ru подразумевает, что вы прочитали и согласны с пользовательским соглашением http://www.mathnet.ru/rus/agreement

Параметры загрузки:

IP : 107.22 .136 .117

26 апреля 2023 г., 10:38:48 
ТЕОРЕТИЧЕСКАЯ

И МАТЕМАТИЧЕСКАЯ

ФИЗИКА

Том 113, № 1

октябрь, 1997

М. А. Васильев* , И. В. Тютин*

\section{ЛАГРАНЖЕВО ОПИСАНИЕ НЕПРИВОДИМЫХ МАССИВНЫХ ПОЛЕЙ ПРОИЗВОЛЬНОГО СПИНА В РАЗМЕРНОСТИ $2+1$}

Дано лагранжево описание свободных массивных полей, образующих неприводимое представление группы Пуанкаре произвольного целого и полуцелого спина в пространстве-времени размерности 3 . Обсуждается взаимосвязь рассматриваемой теории с уравнениями самодуальности в четырех измерениях.

\section{1. ВВЕДЕНИЕ}

Цель настоящей работы - дать лагранжево описание свободных массивных полей, образующих неприводимое представление группы Пуанкаре произвольного целого (бозоны) или полуцелого (фермионы) спина в пространстве-времени размерности 3 . Интерес к моделям в пространстве малой размерности связан прежде всего с тем, что, будучи относительнно простыми, такие теории служат полигоном для изучения более реалистических теорий в пространствах большего числа измерений. Кроме того, можно надеяться на то, что эти модели могут описывать физические системы типа пленок.

K настояшему моменту имеется большое число работ начиная с классической работы Фирца и Паули [1], в которых построены калибровочно-инвариантные действия для безмассовых полей произвольного спина в произвольной размерности [2] и действия для массивных полей, отвечающих полностью симметричным представлениям малой группы в размерностях $D \geq 4$ [3]. Отметим работу [4], в которой продемонстрировано, что действия для массивных полей в размерности $D$ могут быть получены размерной редукцией действий для безмассовых полей в размерности $D+1$. При этом автоматически возникают дополнительные поля, без которых невозможно построение локального действия. Прямолинейное использование этой процедуры для построения действия массивных полей в размерности 3 путем редукции калибровочно-инвариантного действия в размерности 4 [5-9] сталкивается со следующей проблемой. Калибровочно-инвариантные действия в размерности 4 описывают поля с двумя значениями спиральности (эти поля можно интерпретировать либо как майорановские поля с двумя значениями спиральности, либо как вейлевские поля и антиполя, каждое с одним значением спиральности). При редукции в трехмерное пространство возникает действие, которое описывает поле с двумя значениями $(2+1)$-мерного спина, оказываюшееся, следовательно, приводимым

* Физический институт им. П.Н. Лебедева, Москва, Россия 
представлением $(2+1)$-мерной группы Пуанкаре [10]. Поскольку в размерности 4 невозможно построить локальное действие, описываюшее самодуальное поле с одним значением спиральности [11] (майорано-вейлевское поле), после размерной редукции $4 \rightarrow 3$ необходимо выполнить дополнительную редукцию, вдвое уменьшаюшую число физических компонент, если мы хотим, чтобы физическая система отвечала неприводимому представлению $(2+1)$-мерной группы Пуанкаре.

До сих пор в литературе были предложены действия для неприводимых массивных полей в $(2+1)$-мерном пространстве со спинами $s=1[12-14], s=3 / 2[15,16]$, $s=2[14,17]$ и $s=5 / 2[18,19]$. Формализм, применяемый в данной работе для построения лагранжева описания произвольных неприводимых представлений $(2+1)$-мерной группы Пуанкаре, связан с редукцией в размерность $2+1$ тетрадоподобных переменных, введенных для описания безмассовых полей произвольного целого [8] и полуцелого [8,9] спинов в $3+1$ измерениях. В случае спинов $3 / 2,2$ и $5 / 2$ мы воспроизводим результаты работ $[16,14,18]$.

План работы следующий. Раздел 2 посвящен описанию используемых обозначений. В разделе 3 построены действия (первого порядка по производным) для бозонных полей произвольного целого спина $s,|s| \geq 2$, а в разделе 4 то же самое сделано для фермионных полей произвольного полуцелого спина $s,|s| \geq 3 / 2$. В разделе 5 рассмотрены ограничения на параметры модели (выбор знаков перед кинетическим и массовым членами в действии), следующие из требования положительности энергии и нормы состояний. В разделе 6 для бозонного случая цельх $s$ построено действие второго порядка по производным, описывающее сразу оба неприводимых представления $(2+1)$-мерной группы Пуанкаре с фиксированным значением $|s|$.

\section{2. ОБОЗНАЧЕНИЯ И ПОСТАНОВКА ЗАДАЧИ}

В качестве полевых переменных мы будем использовать симметричные по всем спинорным индексам поля $\lambda_{\alpha(n)}^{n} \equiv \lambda_{\alpha_{1} \ldots \alpha_{n}}^{n}$ и $h_{\mu \mid \alpha(n)}^{n} \equiv h_{\mu \mid \alpha_{1} \ldots \alpha_{n}}^{n}, \mu, \nu, \ldots=0,1,2$; $\alpha_{1}, \ldots, \alpha_{n}, \beta, \ldots=1,2^{1)}$. Для этих полей будут также использоваться обозначения $\lambda^{n}$ и $h_{\mu}^{n}$. Поднимание и опускание векторных индексов производится обычным образом с помощью метрических тензоров $\eta^{\mu \nu}=\eta_{\mu \nu}=\operatorname{diag}(+,-,-)$. Поднимание и опускание спинорных индексов производится с помошью $\varepsilon_{\alpha \beta}=-\varepsilon_{\beta \alpha}$ и $\varepsilon^{\alpha \beta}=-\varepsilon^{\beta \alpha}$, причем $\varepsilon_{12}=\varepsilon^{12}=1$ :

$$
A_{\alpha}=A^{\beta} \varepsilon_{\beta \alpha}, \quad A^{\alpha}=\varepsilon^{\alpha \beta} A_{\beta}, \quad A^{\alpha} B_{\alpha}=-A_{\alpha} B^{\alpha} .
$$

Преобразование Лоренца для полей $h_{\mu}^{n}$ и $\lambda^{n}$, отвечающее преобразованию координат $\delta x^{\mu}=\omega^{\mu \nu} x_{\nu}, \omega^{\mu \nu}=-\omega^{\nu \mu}$, имеет вид

$$
\begin{aligned}
\delta h_{\mu \mid \alpha(n)}^{n}= & \frac{i}{2} \omega^{\nu \sigma}\left(M_{\nu \sigma} h^{n}\right)_{\mu \mid \alpha(n)}=\omega^{\nu \sigma} x_{\nu} \partial_{\sigma} h_{\mu \mid \alpha(n)}^{n}+ \\
& +\omega_{\mu \nu} h^{n \nu \mid}{ }_{\alpha(n)}-\frac{n}{4} \omega^{\nu \sigma} \varepsilon_{\nu \sigma \rho} e^{\rho \mid}{ }_{\alpha}^{\beta} h_{\mu \mid \alpha(n-1) \beta}^{n}, \\
\delta \lambda_{\alpha(n)}^{n}= & \omega^{\nu \sigma} x_{\nu} \partial_{\sigma} \lambda_{\alpha(n)}^{n}-\frac{n}{4} \omega^{\nu \sigma} \varepsilon_{\nu \sigma \rho} e^{\rho \mid}{ }_{\alpha}^{\beta} \lambda_{\alpha(n-1) \beta}^{n} .
\end{aligned}
$$

\footnotetext{
1) Здесь и ниже греческие индексы из первой половины алфавита являются спинорными, $\alpha, \beta, \ldots=1,2 ;$ из второй половины алфавита - векторными, $\mu, \nu, \ldots=0,1,2$.
} 
Здесь $\partial_{\mu} \equiv \partial / \partial x^{\mu}, \varepsilon_{\mu \nu \sigma}-$ полностью антисимметричный тензор, $\varepsilon_{012}=\varepsilon^{012}=1$, $e^{\mu \mid}{ }_{\alpha \beta}$ - набор трех вещественных симметричных матриц, свойства которых определяются соотношением

$$
e^{\mu \mid}{ }_{\alpha \gamma} e^{\nu \mid}{ }_{\beta}^{\gamma}=\eta^{\mu \nu} \varepsilon_{\alpha \beta}-\varepsilon^{\mu \nu \sigma} e_{\sigma \mid \alpha \beta}
$$

и которые удовлетворяют следующим полезным тождествам:

$$
e_{\mu \mid \alpha(2)} e^{\mu \mid \beta(2)}=2 \delta_{\alpha}^{\beta} \delta_{\alpha}^{\beta}, \quad \varepsilon^{\mu \nu \sigma} e_{\nu \mid \alpha(2)} e_{\sigma \mid}^{\beta(2)}=-2 \delta_{\alpha}^{\beta} e^{\mu \mid}{ }_{\alpha}^{\beta}
$$

(в разделе 6 будет использована специальная реализация, в которой $e^{0 \mid}{ }_{\alpha \beta}=\delta_{\alpha \beta}$ ). Примем следуюшее соглашение [20]:

$$
A_{\alpha(k) \beta(l)}^{k+l} B_{\alpha(m) \gamma(n)}^{m+n} \equiv \frac{1}{C_{k+m}^{k}} \sum A_{\alpha_{i_{1}} \ldots \alpha_{i_{k}} \beta(l)}^{k+l} B_{\alpha_{i_{k+1}} \ldots \alpha_{i_{k+m}} \gamma(n)}^{m+n},
$$

суммирование ведется по всем $C_{k+m}^{k}$ сочетаниям индексов $\alpha_{i}, A^{k+l}$ и $B^{m+n}$ симметричны по своим спинорным индексам, кроме того,

$$
\begin{gathered}
A^{n+m \alpha(n) \beta(m)} B_{\alpha(n+k) \gamma(l)}^{n+k+l} \equiv \sum_{\alpha_{i}} A^{n+m \alpha_{1} \ldots \alpha_{n} \beta(m)} B_{\alpha_{1} \ldots \alpha_{n} \alpha(k) \gamma(l)}^{n+k+l}, \\
A^{n+m \alpha(n) \beta(m)} B_{\alpha\left(n_{1}\right) \gamma(k)}^{n_{1}+k} C_{\alpha\left(n-n_{1}\right) \delta(l)}^{n-n_{1}+l} \equiv A^{n+m \alpha(n) \beta(m)}\left(B_{\alpha\left(n_{1}\right) \gamma(k)}^{n_{1}+k} C_{\alpha\left(n-n_{1}\right) \delta(l)}^{n-n_{1}+l}\right) .
\end{gathered}
$$

Оператор Казимира группы Пуанкаре (скаляр Паули-Любаньского)

$$
W=-\frac{i}{2} \varepsilon^{\mu \nu \sigma} M_{\nu \sigma} \partial_{\mu}
$$

действует на $h_{\mu}^{n}$ по правилу

$$
\left(W h^{n}\right)_{\mu \mid \alpha(n)}=\varepsilon_{\mu}^{\nu \sigma} \partial_{\nu} h_{\sigma \mid \alpha(n)}^{n}+\frac{n}{2} e^{\sigma \mid}{ }_{\alpha}^{\beta} \partial_{\sigma} h_{\mu \mid \alpha(n-1) \beta}^{n} .
$$

Его собственные значения $m s$ определяют спин состояния $s$ относительно группы Пуанкаре; $m$ - масса состояния, которую мы всегда будем считать положительной.

Поле $h_{\mu}^{N}$ описьвает только один (старший) спин $\pm(N+2) / 2$, если оно удовлетворяет условию

$$
e^{\mu \mid}{ }_{\beta}^{\gamma} h_{\mu \mid \alpha(N-1) \gamma}^{N}=0
$$

(которое означает, что поле $\tilde{h}_{\beta_{1} \beta_{2} \alpha_{1} \ldots \alpha_{N}}^{N+2} \equiv(1 / 2) e^{\mu \mid}{ }_{\beta_{1} \beta_{2}} h_{\mu \mid \alpha_{1} \ldots \alpha_{N}}^{N}$ симметрично по всем $N+2$ спинорным индексам) и, например,

$$
\varepsilon_{\mu}^{\nu \sigma} \partial_{\nu} h_{\sigma \mid \alpha(N)}^{N}= \pm m h_{\mu \mid \alpha(N)}^{N}
$$

(последнее равенство означает, что поле $\tilde{h}^{N+2}$ удовлетворяет уравнению Дирака по каж дому спинорному индексу).

Наша задача состоит в нахождении экстремального принципа, приводяшего к уравнениям (4), (5) и, быть может, к совокупности динамически тривиальных уравнений для вспомогательных полей. 


\section{3. БОЗОННЫЕ ПОЛЯ}

В этом разделе будет построено действие для бозонных полей произвольного целого спина $s,|s|=(N+2) / 2 \geq 2, N \geq 2$ четное. Начнем с действия

$$
\begin{aligned}
S_{\mathrm{Б}}= & \frac{1}{2} \sum_{n=0}^{N} \xi_{n} \varepsilon^{\mu \nu \sigma} h_{\mu \mid}^{n \alpha(n)} \partial_{\nu} h_{\sigma \mid \alpha(n)}^{n}+ \\
& +\frac{m}{2} \sum_{n=2}^{N} a_{n} \varepsilon^{\mu \nu \sigma} h_{\mu \mid}^{n \alpha(n-1) \beta} e_{\nu \mid \beta^{\gamma}} h_{\sigma \mid \alpha(n-1) \gamma}^{n}+ \\
& +m \sum_{n=2}^{N} b_{n} \varepsilon^{\mu \nu \sigma} h_{\mu \mid}^{n \alpha(n)} e_{\nu \mid \alpha(2)} h_{\sigma \mid \alpha(n-2)}^{n-2}+ \\
& +m \sum_{n=0}^{N-2} \lambda_{\alpha(n+2)}^{n+2} e^{\mu \mid \alpha(2)} h_{\mu \mid}^{n \alpha(n)}
\end{aligned}
$$

где суммирование выполняется по четным $n$. Бозонные поля $h_{\mu}^{n}, \lambda^{n}$ действительные. Из условия вешественности действия следует, что коэффициенты $\xi_{n}, a_{n}, b_{n}$ должны быть вешественными. Мы будем считать, что $\xi_{n}= \pm 1$, чего всегда можно добиться подходящей нормировкой полей. Действие (6) инвариантно относительно преобразований Лоренца (1), (2). Задача состоит в том, чтобы подобрать коэффициенты $\xi_{n}, a_{n}, b_{n}$ таким образом, чтобы лишние компоненты поля $h_{\mu}^{N}$ (включая один из двух старших спинов), а также все поля $h_{\mu}^{n}, 0 \leq n \leq N-2$, и $\lambda^{n}, 2 \leq n \leq N$, обрашались в нуль на уравнениях движения.

Вариация действия по $\lambda^{n}$ дает уравнения связей

$$
e_{\alpha(2)}^{\mu \mid} h_{\mu \mid \alpha(n)}^{n}=0, \quad 0 \leq n \leq N-2
$$

Из (7) при $n=0$ следует

$$
h_{\mu}^{0}=0
$$

Вариация действия по $h_{\mu}^{n}$ дает уравнения движения

$$
\begin{aligned}
& \xi_{n} \varepsilon^{\mu \nu \sigma} \partial_{\nu} h_{\sigma \mid \alpha(n)}^{n}+\left(1-\delta_{n, 0}\right) m a_{n} \varepsilon^{\mu \nu \sigma} e_{\nu \mid \alpha}{ }^{\beta} h_{\sigma \mid \alpha(n-1) \beta}^{n}+ \\
& +\left(1-\delta_{n, 0}\right) m b_{n} \varepsilon^{\mu \nu \sigma} e_{\nu \mid \alpha(2)} h_{\sigma \mid \alpha(n-2)}^{n-2}- \\
& -\left(1-\delta_{n, N}\right) m b_{n+2} \varepsilon^{\mu \nu \sigma} e_{\nu \mid}^{\alpha(2)} h_{\sigma \mid \alpha(n+2)}^{n+2}+ \\
& +\left(1-\delta_{n, N}\right) m e^{\mu \mid \alpha(2)} \lambda_{\alpha(n+2)}^{n+2}=0 \text {. }
\end{aligned}
$$

Возьмем дивергенцию левой части (9) по $\partial_{\mu}$ и в полученных уравнениях исключим производные всех функций $h_{\sigma}^{n}$ с помошью (9). При каждом $n$ в возникаюших уравнениях могут появиться величины $h_{\sigma}^{n+4}, h_{\sigma}^{n+2}, h_{\sigma}^{n}, h_{\sigma}^{n-2}, h_{\sigma}^{n-4}$. Коэффииценты перед $h_{\sigma}^{n+4}$ и 
$h_{\sigma}^{n-4}$ тождественно равны нулю из-за свойств матриц $e_{\mu}$ и антисимметризации по векторным индексам. Слагаемое с $h_{\sigma}^{n-2}$ равно нулю в силу уравнений связей $(7)^{2)}$. В результате получаем после некоторых преобразований

$$
\begin{gathered}
2 \xi_{2} a_{2} b_{2} e^{\mu \mid \alpha(2)} h_{\mu \mid \alpha(2)}^{2}=\frac{1}{m} e^{\mu \mid \alpha(2)} \partial_{\mu} \lambda_{\alpha(2)}^{2}, \\
b_{n+2}\left(\xi_{n} a_{n}-\frac{n+4}{n+2} \xi_{n+2} a_{n+2}\right) e^{\sigma \mid \alpha(2)} h_{\sigma \mid \alpha(n+2)}^{n+2}+ \\
+\left(\frac{2}{n} \xi_{n} a_{n}^{2}+2 \xi_{n-2} b_{n}^{2}-\frac{2 n(n+3)}{(n+1)(n+2)} \xi_{n+2} b_{n+2}^{2}\right) e^{\sigma \mid}{ }_{\alpha}^{\beta} h_{\sigma \mid \alpha(n-1) \beta}^{n}+ \\
+2 \xi_{n-2} b_{n} \lambda_{\alpha(n)}^{n}+\frac{1}{m}\left(1-\delta_{n, N}\right) e^{\mu \mid \alpha(2)} \partial_{\mu} \lambda_{\alpha(n+2)}^{n+2}=0, \quad 2 \leq n \leq N,
\end{gathered}
$$

где по определению $b_{N+2}=0$.

Потребуем, чтобы коэффициенты перед $h_{\sigma}^{n+2}$ и $h_{\sigma}^{n}$ в $(11)$ обрашались в нуль:

$$
\begin{aligned}
& \xi_{n} a_{n}-\frac{n+4}{n+2} \xi_{n+2} a_{n+2}=0, \quad 2 \leq n \leq N \\
& \frac{1}{n} \xi_{n} a_{n}^{2}+\xi_{n-2} b_{n}^{2}-\frac{n(n+3)}{(n+1)(n+2)} \xi_{n+2} b_{n+2}^{2}=0, \quad 2 \leq n \leq N .
\end{aligned}
$$

Решение этих уравнений имеет вид

$$
a_{n}=\frac{N+2}{n+2} \xi_{N} \xi_{n}(-1)^{(N+2) / 2} \theta, \quad b_{n}^{2}=-\xi_{n-2} \xi_{n} \frac{(N+2)^{2}-n^{2}}{4 n(n+1)}, \quad \theta^{2}=1,
$$

где выбрана нормировка $a_{N}=(-1)^{(N+2) / 2} \theta$, что всегда может быть достигнуто переопределением параметра $m$. В разделе 5 будет показано, что условие положительности энергии и нормы состояний выполняется только при $\theta=1$, что мы и примем ниже. Условие $b_{n}^{2}>0$ дает $\xi_{n-2} \xi_{n}=-1$, т.е.

$$
\xi_{n}=(-1)^{(n+2) / 2} \xi, \quad \xi^{2}=1,
$$

так что окончательно имеем

$$
\begin{aligned}
& \xi_{n}=(-1)^{(n+2) / 2} \xi, \quad a_{n}=(-1)^{(n+2) / 2} \frac{N+2}{n+2}, \\
& b_{n}=\frac{(-1)^{(n+2) / 2}}{2} \sqrt{\frac{(N+2)^{2}-n^{2}}{n(n+1)}} .
\end{aligned}
$$

Любой другой выбор знаков для $b_{n}$ может быть скомпенсирован простым переопределением полей.

Полагая $n=N$ в (11), получаем

$$
\lambda_{\alpha(N)}^{N}=0
$$

\footnotetext{
${ }^{2)}$ Отметим, что здесь реально необходимо лишь одно из уравнений связей, именно (8). При $n \geq 4$ коэффициент перед $h_{\sigma}^{n-2}$ исчезает, если взять $a_{n}$ из (12).
} 
Полагая там же $n=N-2$ и учитьвая (13), получаем

$$
\lambda_{\alpha(N-2)}^{N-2}=0
$$

и т.д.

Окончательно из (11) следует, что на уравнениях движения все лагранжевы множители равны нулю:

$$
\lambda_{\alpha(n)}^{n}=0, \quad n=2, \ldots, N .
$$

Перейдем к изучению уравнений для $h_{\mu}^{n}$.

Пусть $N=2$. Уравнение движения (9) при $n=0$ (с учетом $(8))$ и уравнение $(10)$ дают

$$
\varepsilon^{\mu \nu \sigma} e_{\nu \mid}^{\alpha(2)} h_{\sigma \mid \alpha(2)}^{2}=0, \quad e^{\mu \mid \alpha(2)} h_{\mu \mid \alpha(2)}^{2}=0
$$

что эквивалентно условию (4). Уравнение движения (9) при $n=2$ имеет вид

$$
\varepsilon^{\mu \nu \sigma} \partial_{\nu} h_{\sigma \mid \alpha(2)}^{2}+\xi m \varepsilon^{\mu \nu \sigma} e_{\nu \mid \alpha}^{\beta} h_{\sigma \mid \alpha \beta}^{2}=0
$$

что эквивалентно (5).

Пусть $N>2$. Уравнение движения (9) при $n=0$ (с учетом (8)), уравнение связи $(7)$ при $n=2$ и уравнение (10) дают

$$
\varepsilon^{\mu \nu \sigma} e_{\nu \mid}^{\alpha(2)} h_{\sigma \mid \alpha(2)}^{2}=0, \quad e^{\mu \mid}{ }_{\alpha(2)} h_{\mu \mid \alpha(2)}^{2}=0, \quad e^{\mu \mid \alpha(2)} h_{\mu \mid \alpha(2)}^{2}=0,
$$

откуда, как нетрудно убедиться, следует

$$
h_{\mu \mid \alpha(2)}^{2}=0 .
$$

Далее предположим, что мы проверили, что все $h_{\mu}^{k}, 2 \leq k \leq n_{0}<N-2$, равны нулю на уравнениях движения. Тогда уравнение движения (9) при $n=n_{0}$ и уравнение связи (7) при $n=n_{0}+2$ дают

$$
\varepsilon^{\mu \nu \sigma} e_{\nu \mid}^{\alpha(2)} h_{\sigma \mid \alpha\left(n_{0}+2\right)}^{n_{0}+2}=0, \quad e_{\alpha(2)}^{\mu \mid} h_{\mu \mid \alpha\left(n_{0}+2\right)}^{n_{0}+2}=0,
$$

откуда следует

$$
h_{\mu \mid \alpha\left(n_{0}+2\right)}^{n_{0}+2}=0 .
$$

Итак, уравнение движения (9) при $n \leq N-4$, уравнения связи (8) и (7) и уравнение (10) дают

$$
h_{\mu \mid \alpha(n)}^{n}=0, \quad n=0, \ldots, N-2 .
$$

Наконец, из уравнения движения (9) при $n=N-2$ и $n=N$ можно получить

$$
\begin{gathered}
\varepsilon^{\mu \nu \sigma} e_{\nu \mid}{ }^{\alpha(2)} h_{\sigma \mid \alpha(N)}^{N}=0, \\
\varepsilon^{\mu \nu \sigma} \partial_{\nu} h_{\sigma \mid \alpha(N)}^{N}+\xi m \varepsilon^{\mu \nu \sigma} e_{\nu \mid \alpha}^{\beta} h_{\sigma \mid \alpha(N-1) \beta}^{N}=0 .
\end{gathered}
$$

Условие (14) при $N \geq 3$ эквивалентно условию (4). В силу этого условия уравнения (15) могут быть переписаны в эквивалентных формах:

$$
\begin{gathered}
\varepsilon_{\mu}{ }^{\nu \sigma} \partial_{\nu} h_{\sigma \mid \alpha(N)}^{N}-\xi m h_{\mu \mid \alpha(N)}^{N}=0, \\
e^{\nu \mid}{ }_{\beta}^{\gamma} \partial_{\nu} h_{\mu \mid \alpha(N-1) \gamma}^{N}-\xi m h_{\mu \mid \beta \alpha(N-1)}^{N}=0 .
\end{gathered}
$$


Из этих представлений следуют равенства

$$
\begin{gathered}
\left(\square+m^{2}\right) h_{\mu \mid \alpha(N)}^{N}=0, \quad \square=\partial_{0}^{2}-\partial_{1}^{2}-\partial_{2}^{2}, \\
\left(W h^{N}\right)_{\mu \mid \alpha(N)}=m\left(\xi \frac{N+2}{2}\right) h_{\mu \mid \alpha(N)}^{N} .
\end{gathered}
$$

Таким образом, параметр $m$ совпадает с массой покоя, а из (17) следует, что поле $h_{\mu}^{N}$ имеет спин $\xi(N+2) / 2$.

Итак, установлено, что действие (6) при выборе параметров (12) описывает неприводимое представление $(2+1)$-мерной группы Пуанкаре спина $\xi(N+2) / 2$.

\section{4. ФЕРМИОННЫЕ ПОЛЯ}

В этом разделе будет построено действие для фермионных полей произвольного полуцелого спина $s,|s|=(N+2) / 2 \geq 3 / 2$, где $N \geq 1$ нечетное:

$$
\begin{aligned}
S_{\Phi}= & \frac{i}{2} \sum_{n=1}^{N} \xi_{n} \varepsilon^{\mu \nu \sigma} h_{\mu \mid}^{n \alpha(n)} \partial_{\nu} h_{\sigma \mid \alpha(n)}^{n}+ \\
& +\frac{i \varepsilon m}{2} \sum_{n=1}^{N} a_{n} \varepsilon^{\mu \nu \sigma} h_{\mu \mid}^{n \alpha(n-1) \beta} e_{\nu \mid \beta}^{\gamma} h_{\sigma \mid \alpha(n-1) \gamma}^{n}+ \\
& +i m \sum_{n=3}^{N} b_{n} \varepsilon^{\mu \nu \sigma} h_{\mu \mid}^{n \alpha(n)} e_{\nu \mid \alpha(2)} h_{\sigma \mid \alpha(n-2)}^{n-2}+ \\
& +i m \sum_{n=1}^{N-2} \lambda_{\alpha(n+2)}^{n+2} e^{\mu \mid \alpha(2)} h_{\mu \mid}^{n \alpha(n)}
\end{aligned}
$$

и суммирование вьполняется по нечетным $n$. Фермионные поля $h_{\mu}^{n}$ и $\lambda^{n}$ действительные, коэффициенты $a_{n}, b_{n}$ вешественны, $\xi_{n}= \pm 1, \varepsilon= \pm 1$.

Уравнения связей имеют вид

$$
e_{\alpha(2)}^{\mu \mid} h_{\mu \mid \alpha(n)}^{n}=0, \quad n=1, \ldots, N-2 .
$$

Уравнения движения аналогичны уравнениям движения для бозонных полей:

$$
\begin{aligned}
\xi_{n} \varepsilon^{\mu \nu \sigma} \partial_{\nu} h_{\sigma \mid \alpha(n)}^{n}+\varepsilon m a_{n} \varepsilon^{\mu \nu \sigma} e_{\nu \mid \alpha}{ }^{\beta} h_{\sigma \mid \alpha(n-1) \beta}^{n}+\left(1-\delta_{n, 1}\right) m b_{n} \varepsilon^{\mu \nu \sigma} e_{\nu \mid \alpha(2)} h_{\sigma \mid \alpha(n-2)}^{n-2}- \\
-\left(1-\delta_{n, N}\right) m b_{n+2} \varepsilon^{\mu \nu \sigma} e_{\nu \mid}^{\alpha(2)} h_{\sigma \mid \alpha(n+2)}^{n+2}-\left(1-\delta_{n, N}\right) m e^{\mu \mid \alpha(2)} \lambda_{\alpha(n+2)}^{n+2}=0 .
\end{aligned}
$$

Как и в бозонном случае, возьмем дивергенцию левой части (20) по $\partial_{\mu}$ и исключим производные всех функций $h_{\sigma}^{n}$ с помощью (20). В возникающих уравнениях при фиксированном $n$ коэффициенты перед $h_{\sigma}^{n+4}$ и $h_{\sigma}^{n-4}$ тождественно равны нулю, а слагаемое с $h_{\sigma}^{n-2}$ равно нулю в силу уравнений связей $(19)^{3)}$, в результате чего после некоторых

\footnotetext{
${ }^{3)}$ Слагаемое с $h_{\sigma}^{n-2}$ исчезает и без использования уравнений связей, поскольку коэффициент перед $h_{\sigma}^{n-2}$ равен нулю одновременно с равенством нулю коэффициента перед $h_{\sigma}^{n+2}$.
} 
преобразований приходим к следующему:

$$
\begin{aligned}
& \varepsilon b_{3}\left(\xi_{1} a_{1}-\frac{5}{3} \xi_{3} a_{3}\right) e^{\sigma \mid \alpha(2)} h_{\sigma \mid \alpha(3)}^{3}+ \\
& \quad+\left(2 \xi_{1} a_{1}^{2}-\frac{4}{3} \xi_{3} b_{3}^{2}\right) e^{\sigma \mid}{ }_{\alpha}^{\beta} h_{\sigma \mid \beta}^{1}-\frac{1}{m} e^{\mu \mid \alpha(2)} \partial_{\mu} \lambda_{\alpha(3)}^{3}=0 \\
& \varepsilon b_{n+2}\left(\xi_{n} a_{n}-\frac{n+4}{n+2} \xi_{n+2} a_{n+2}\right) e^{\sigma \mid \alpha(2)} h_{\sigma \mid \alpha(n+2)}^{n+2}+ \\
& \quad+\left(\frac{2}{n} \xi_{n} a_{n}^{2}+2 \xi_{n-2} b_{n}^{2}-\frac{2 n(n+3)}{(n+1)(n+2)} \xi_{n+2} b_{n+2}^{2}\right) e^{\sigma \mid}{ }_{\alpha}^{\beta} h_{\sigma \mid \alpha(n-1) \beta}^{n}- \\
& \quad-2 \xi_{n-2} b_{n} \lambda_{\alpha(n)}^{n}-\frac{1}{m}\left(1-\delta_{n, N}\right) e^{\mu \mid \alpha(2)} \partial_{\mu} \lambda_{\alpha(n+2)}^{n+2}=0, \quad 3 \leq n \leq N
\end{aligned}
$$

Как и в бозонном случае, по определению $b_{N+2}=0$. Потребуем, чтобы в уравнении $(21)$ обрашался в нуль коэффищиент перед $h_{\sigma}^{3}$, а в уравнениях $(22)$ - коэффициенты перед $h_{\sigma}^{n+2}$ и $h_{\sigma}^{n}$ :

$$
\begin{gathered}
\xi_{n} a_{n}-\frac{n+4}{n+2} \xi_{n+2} a_{n+2}=0, \quad 1 \leq n \leq N \\
\frac{1}{n} \xi_{n} a_{n}^{2}+\xi_{n-2} b_{n}^{2}-\frac{n(n+3)}{(n+1)(n+2)} \xi_{n+2} b_{n+2}^{2}=0, \quad 3 \leq n \leq N .
\end{gathered}
$$

Эти уравнения аналогичны уравнениям бозонного случая и имеют те же решения. Примем нормировку $a_{N}=(-1)^{(N+1) / 2}$. Кроме того, в разделе 5 будет показано, что условие положительности энергии и нормы состояний требует $\xi_{N}=(-1)^{(N+1) / 2}$. В результате получаем

$$
\begin{aligned}
& \xi_{n}=(-1)^{(n+1) / 2}, \quad a_{n}=(-1)^{(n+1) / 2} \frac{N+2}{n+2} \\
& b_{n}=\frac{(-1)^{(n+1) / 2}}{2} \sqrt{\frac{(N+2)^{2}-n^{2}}{n(n+1)}} .
\end{aligned}
$$

Далее, как и в бозонном случае, из уравнений (22) следует, что все лагранжевы множители $\lambda^{n}$ равны нулю, а уравнение (21) принимает вид

$$
e^{\mu \mid}{ }_{\alpha}^{\beta} h_{\mu \mid \beta}^{1}=0
$$

Обратимся к уравнениям для $h_{\mu}^{n}$.

Пусть $N=1$. В этом случае лагранжевы множители отсутствуют, а $h_{\mu \mid \alpha}^{1}$ удовлетворяет уравнению

$$
\varepsilon^{\mu \nu \sigma} \partial_{\nu} h_{\sigma \mid \alpha}^{1}+\varepsilon m \varepsilon^{\mu \nu \sigma} e_{\nu \mid \alpha}^{\beta} h_{\sigma \mid \beta}^{1}=0
$$

Это уравнение и его следствие (24) эквивалентны уравнениям (4), (5).

Пусть $N \geq 3$. Из уравнения связи (19) при $n=1$ и уравнения (24) следует $h_{\mu \mid \alpha}^{1}=0$. Далее, как и в бозонном случае, убеждаемся, что $h_{\mu}^{n}=0,3 \leq n \leq N-2$. Уравнения 
движения (20) при $n=N-2$ дают для $h_{\mu}^{N}$ условие (14), эквивалентное (4), а те же уравнения (20) при $n=N$ дают

$$
\varepsilon^{\mu \nu \sigma} \partial_{\nu} h_{\sigma \mid \alpha(N)}^{N}+\varepsilon m \varepsilon^{\mu \nu \sigma} e_{\nu \mid \alpha}^{\beta} h_{\sigma \mid \alpha(N-1) \beta}^{N}=0,
$$

откуда следует, что $h_{\mu}^{N}$ удовлетворяет уравнению Клейна-Гордона с массой $m$ и описывает спин $\varepsilon(N+2) / 2$.

Отметим, что если факторы $i$ и $\varepsilon$ включить в определение коэффициентов $\xi_{n}, a_{n}$ и $b_{n}$, то параметры бозонного и фермионного действий могут быть записаны едиными формулами:

$$
\begin{gathered}
\tilde{\xi}_{n}=\zeta_{n} \xi, \quad \tilde{a}_{n}=\zeta_{n} \theta \frac{N+2}{n+2}, \quad \tilde{b}_{n}=\frac{\zeta_{n}}{2} \sqrt{\frac{(N+2)^{2}-n^{2}}{n(n+1)}} \\
\zeta_{n}=(-1)^{(n+2) / 2}=\exp \left(i \pi \frac{n+2}{2}\right),
\end{gathered}
$$

где

$$
\tilde{a}_{n}=i \epsilon a_{n}, \quad \tilde{\xi}_{n}=i \xi_{n}, \quad \tilde{b}_{n}=i b_{n}
$$

в фермионном случае и

$$
\tilde{a}_{n}=a_{n}, \quad \tilde{\xi}_{n}=\xi_{n}, \quad \tilde{b}_{n}=b_{n}
$$

в бозонном.

\section{5. ГАМИЛЬТОНОВ ФОРМАЛИЗМ}

В этом разделе будут рассмотрены ограничения на параметры модели, следуюшие из условия положительности энергии и нормы состояний. Как мы видели выше, на уравнениях движения поля $h_{\mu}^{n}, n<N$, все лагранжевы множители $\lambda^{n}$ и некоторые компоненты поля $h_{\mu}^{N}$ равны нулю. Это означает, что в теории имеются связи. Поскольку теория не вырождена (нет калибровочной симметрии), связи могут быть только второго рода. Как показано в [21], связи второго рода можно подставлять в исходное действие, в результате чего возникает эквивалентная теория. Мы воспользуемся этим обстоятельством. Кроме того, в силу лоренщ-инвариантности для наших целей достаточно рассмотреть компоненты $h_{\mu}^{N}$ при нулевом импульсе (в системе покоя). Уравнения движения (15), (25) с учетом связей (14) (или эквивалентно (16)) дают при $\mu=0$

$$
h_{0 \mid \alpha(N)}^{N}=0 .
$$

Введем поле $(1 / 2) e_{\alpha_{1} \alpha_{2}}^{\mu \mid} h_{\mu \mid \alpha_{3} \ldots \alpha_{N+2}}^{N} \equiv \tilde{h}_{\alpha(N+2)}^{N+2}$, которое симметрично по всем $N+2$ спинорным индексам в силу условия (14) (или эквивалентно (4)). Уравнение (26) означает, что поле $\tilde{h}^{N+2}$ бесследово (используется реализация матриц $e_{\mu}$, в которой $e_{0 \mid \alpha \beta}=\delta_{\alpha \beta}$ ):

$$
\tilde{h}_{11 \alpha(N)}^{N+2}+\tilde{h}_{22 \alpha(N)}^{N+2}=0 .
$$

Таким образом, $\tilde{h}^{N+2}$ имеет всего две независимые компоненты:

$$
\tilde{h}_{1 \ldots 1}^{N+2} \equiv 2^{-(N+2) / 2} q_{1}, \quad \tilde{h}_{21 \ldots 1}^{N+2} \equiv 2^{-(N+2) / 2} q_{2} .
$$


Выразим действия (6) и (18) через переменные $q_{1}$ и $q_{2}$, пользуясь соотношением

$$
h_{\mu \mid \alpha(N)}^{N}=e_{\mu \mid}^{\alpha(2)} \tilde{h}_{\alpha(N+2)}^{N+2} .
$$

Бозонный случай. Используя равенства $\xi_{N}=(-1)^{(N+2) / 2} \xi$ и $a_{N}=(-1)^{(N+2) / 2} \theta$, после несложных преобразований получаем

$$
S_{\mathrm{Б}}=\xi q_{2} \dot{q}_{1}-\frac{\theta m}{2}\left(q_{1}^{2}+q_{2}^{2}\right)
$$

Из этого выражения видно, что $\xi$ может иметь любой знак, в то время как $\theta$ должно быть положительным.

Фермионный случай. Возьмем $\xi_{N}=(-1)^{(N+1) / 2} \xi, \xi= \pm 1$ и $a_{N}=(-1)^{(N+1) / 2}$, тогда

$$
S_{\phi}=\frac{i \xi}{2}\left(q_{1} \dot{q}_{1}+q_{2} \dot{q}_{2}\right)-i \varepsilon m q_{1} q_{2} .
$$

Удобно перейти к новой комплексной переменной $\eta$ :

$$
\eta=\frac{1}{\sqrt{2}}\left(q_{1}+i \xi \varepsilon q_{2}\right), \quad q_{1}=\frac{1}{\sqrt{2}}\left(\eta+\eta^{\dagger}\right), \quad q_{2}=\frac{\xi \varepsilon}{i \sqrt{2}}\left(\eta-\eta^{\dagger}\right),
$$

в терминах которой действие выглядит следующим образом:

$$
S_{\phi}=\xi\left(i \eta^{\dagger} \dot{\eta}-m \eta^{\dagger} \eta\right)
$$

Это выражение явно показывает, что $\varepsilon$ может иметь любой знак, в то время как для $\xi$ допустимо только одно значение $\xi=1$.

\section{6. КВАДРИРОВАНИЕ}

Выше мы построили действия для неприводимых представлений $(2+1)$-мерной группы Пуанкаре, которые в бозонном случае можно записать в виде

$$
S_{\mathrm{Б}}=\frac{\xi}{2} \varphi D_{N} \varphi+\frac{1}{2} \varphi M_{N} \varphi
$$

а в фермионном случае - в виде

$$
S_{\text {ф }}=\frac{i}{2} \psi D_{N} \psi+\frac{i \varepsilon}{2} \psi M_{N} \psi
$$

где $\varphi(\psi)$ - набор всех полей $h_{\mu}^{n}, \lambda^{n}$ в бозонном (фермионном) случае, $D_{N}$ - однородньй симметричньй (антисимметричный) дифференциальный оператор первого порядка, $M_{N}$ - симметричная (антисимметричная) матрица, не содержащая производных. Матрица $M_{N}$ обратима и в бозонном, и в фермионном случаях (в то время как оператор $D_{N}$ необратим). Естественно предположить, что операторы $D_{N} \pm M_{N}$ являются "квадратными корнями" из некоторого дифференциального оператора второго порядка, описываюшего поле с двумя значениями спина, по крайней мере в бозонном случае. Как говорилось во введении, такие операторы естественно возникают при редукции четырехмерных калибровочных действий. 
Ниже будет построено действие для бозонов, имеющее второй порядок по производным и описывающее поля спинов $\pm s$, причем оператор второго порядка получается квадрированием оператора первого порядка. Начнем с действия

$$
S=\frac{1}{2} \varphi\left(-D_{N}+M_{N}\right) R\left(D_{N}+M_{N}\right) \varphi=-\frac{1}{2} \varphi D_{N} R D_{N} \varphi+\frac{1}{2} \varphi M_{N} R M_{N} \varphi
$$

где $R$ - неособенная симметричная матрица. Для того чтобы уравнения движения имели вид

$$
\left(-D_{N}+M_{N}\right) R\left(D_{N}+M_{N}\right) \varphi=0
$$

должно выполняться условие

$$
M_{N} R D_{N}-D_{N} R M_{N}=0 .
$$

Нетрудно видеть, что в этом случае действие (27) описывает поля двух спинов $\pm(N+2) / 2$. Условию (28) можно удовлетворить, выбирая матрицу $R$ равной $M_{N}^{-1}$. Тогда действие (27) принимает вид

$$
S=-\frac{1}{2} \varphi D_{N} M_{N}^{-1} D_{N} \varphi+\frac{1}{2} \varphi M_{N} \varphi
$$

и можно сказать, что операторы первого порядка в действии (6) (и (18)) получаются “извлечением квадратного корня" из оператора второго порядка в действии (29).

Эквивалентное действие получается, если ввести дополнительные поля $\omega$ того же типа, что и $\varphi$ :

$$
S^{\prime}=\frac{1}{2} \varphi M_{N} \varphi+\frac{1}{2} \omega M_{N} \omega+\omega D_{N} \varphi
$$

Делая в этом действии замену

$$
\varphi=\frac{1}{\sqrt{2}}\left(\varphi_{1}+\varphi_{2}\right), \quad \omega=\frac{1}{\sqrt{2}}\left(\varphi_{1}-\varphi_{2}\right),
$$

получаем

$$
S^{\prime}=\frac{1}{2} \varphi_{1}\left(D_{N}+M_{N}\right) \varphi_{1}+\frac{1}{2} \varphi_{2}\left(-D_{N}+M_{N}\right) \varphi_{2}
$$

Таким образом, поле $\varphi_{1}$ описывает спин $(N+2) / 2$, а поле $\varphi_{2}-$ спин $-(N+2) / 2$.

Отметим, что в случае фермионов действие

$$
S=-\frac{\xi}{2} \psi D_{N} M_{N}^{-1} D_{N} \psi+\frac{\xi}{2} \psi M_{N} \psi, \quad \xi= \pm 1
$$

являющееся аналогом бозонного действия (29), также описывает два спина $\pm(N+2) / 2$. Однако при любом $\xi$ оно неприемлемо по физическим соображениям. В этом легче всего убедиться, перейдя к эквивалентному действию

$$
\begin{aligned}
S^{\prime}=\frac{\xi}{2} \psi M_{N} \psi+\frac{\xi}{2} \eta M_{N} \eta+\eta D_{N} \psi & =\frac{\xi}{2} \psi_{1}\left(D_{N}+M_{N}\right) \psi_{1}+\frac{\xi}{2} \psi_{2}\left(-D_{N}+M_{N}\right) \psi_{2}, \\
\psi=\frac{1}{\sqrt{2}}\left(\psi_{1}+\psi_{2}\right), \quad \eta & =\frac{1}{\sqrt{2}}\left(\psi_{1}-\psi_{2}\right) .
\end{aligned}
$$


Видно, что при любом $\xi$ кинетический член одного из фермионов имеет неправильный знак.

Отметим, что столь простое разложение действия для двух поляризаций в сумму действий для независимых компонент оказывается возможным благодаря введению адекватного набора вспомогательных полей, найденных в настоящей работе. При этом поля $\omega$ (точнее их часть, ассоциированная с 1-формами $h_{\mu \mid \alpha(n)}^{n}$ ) могут трактоваться как аналог лоренцевой связности в триадной формулировке гравитации.

\section{7. ЗАКЛЮЧИТЕЛЬНЫЕ ЗАМЕЧАНИЯ}

Описанные в работе динамические системы могут представлять интерес еще и потому, что они тесно связаны с уравнениями самодуальности в $2+2$ измерениях

$$
\varepsilon_{\rho \mu}^{\nu \sigma} \partial_{\nu} h_{\sigma \mid \alpha(N)}^{N}=\partial_{\rho} h_{\mu \mid \alpha(N)}^{N}-\partial_{\mu} h_{\rho \mid \alpha(N)}^{N}
$$

которые переходят в (5) при “компактифицирующей” подстановке

$$
h_{3 \mid \alpha(N)}^{N}=0, \quad \partial_{3} h_{\mu \mid \alpha(N)}^{N}= \pm m h_{\mu \mid \alpha(N)}^{N}
$$

в предположении, что 3-компоненты векторов соответствуют второму временному направлению. Аналогично евклидов вариант (5) может быть получен из евклидовой версии (30).

Отметим, что используемьй в настоящей работе набор полей $h_{\mu \mid \alpha(n)}^{n}(0 \leq n \leq N)$ отвечает разложению полей $h_{\mu \mid \alpha(N / 2) \dot{\alpha}(N / 2)}^{N}\left(N\right.$ четное) и $h_{\mu \mid \alpha((N+1) / 2) \dot{\alpha}((N-1) / 2)}^{N}(N$ нечетное), использовавшихся в [8] для описания четырехмерных безмассовых полей спина $s=N / 2+1$, по неприводимым представлениям диагональной $(2+1)$-мерной группы Лоренца, действующей одновременно на точечные и неточечные спинорные индексы. Любопытно также, что лагранжевы множители $\lambda_{\mu \mid \alpha(n)}^{n}$ имеют ту же структуру по спинорным индексам ( $N \geq n>0)$. Это наталкивает на мысль, что их можно попытаться отождествить с компонентой $(3+1)$-мерного калибровочного поля вдоль “лишнего" времени при размерной редукции некоторой гипотетической самодуальной теории в $(2+2)$-мерном пространстве-времени.

Самодуальные уравнения движения безмассовых полей произвольного спина при $D=4$ могут быть сформулированы не только на линейном, но и на нелинейном уровне [22]. Недавно суперсимметричные самодуальные уравнения для полей произвольного спина изучались в [23]. В контексте полученных результатов было бы интересно вернуться к вопросу о возможности построения теории самодуальных полей произвольного спина в четырехмерной теории на уровне действия.

Также представляло бы интерес построение топологически массивного варианта $(2+1)$-мерной теории массивных полей произвольного спина, обобщаюшего ранее полученные теории для $s=1[13,14], s=3 / 2[15], s=2[14]$ и $s=5 / 2$ [19].

Авторы благодарны С.. Прокушкину за сотрудничество на ранней стадии работы при исследовании динамики спина 3. Работа выполнена при частичной поддержке РФФИ, грант № 96-02-17314, INTAS, грант № 94-2317- $e x t$, и организации NWO Голландии. 


\section{Список литературы}

[1] M. Fierz, W. Pauli. Proc. R. Soc. A. 1939. V. 173. P. 211.

[2] T. Curtright. Phys. Lett. B. 1979. V. 85. P. 219; C.S. Aulakh, I. G. Koh, S. Ouvry. Phys. Lett. B. 1986. V. 173. P. 284; A.K.H. Bengtsson. Phys. Lett. B. 1986. V. 182. P. 321; J. M. F. Labastida. Nucl. Phys. B. 1989. V. 322. P. 185.

[3] W. Rarita, J. Schwinger. Phys. Rev. 1941. V. 60. Р. 61; В. Л. Гинзбург. ЖЭТФ. 1942. T. 12. C. 460; E. C. Фрадкин. ЖЭТФ. 1950. T. 20. C. 27, 211; L. P. S. Singh, C. R. Hagen. Phys. Rev. D. 1974. V. 9. P. 898, 910.

[4] C. Aragone, S. Deser, Z. Yang. Ann. Phys. 1987. V. 179. P. 76.

[5] C. Fronsdal. Phys. Rev. D. 1978. V. 18. P. 3624; 1979. V. 20. P. 848.

[6] J. Fang, C. Fronsdal. Phys. Rev. D. 1978. V. 18. P. 3630; 1980. V. 22. P. 1361.

[7] B. de Wit, D. Z. Freedman. Phys. Rev. D. 1980. V. 21. P. 358.

[8] М. А. Васильев. ЯФ. 1980. Т. 32. С. 855.

[9] C. Aragone, S. Deser. Nucl. Phys. B. 1980. V. 170. [FS1]. P. 329.

[10] B. Binegar. J. Math. Phys. 1982. V. 23. P. 1511.

[11] F. Gliozzi, J. Sherk, D. Olive. Nucl. Phys. B. 1977. V. 122. P. 253; J. Sherk. Extended supersymmetry and extended supergravity theory. In: Recent Development in Gravitation. Eds M. Levy and S. Deser. N. Y.: Plenum Publ. Corp., 1979.

[12] P. K. Townsend, K. Pilch, P. van Nieuwenhuizen. Phys. Lett. B. 1984. V. 136. P. 38.

[13] W. Siegel. Nucl. Phys. B. 1979. V. 156. P. 135; R. Jackiw, S. Templeton. Phys. Rev. D. 1981. V. 23. P. 2291.

[14] S. Deser, R. Jackiw, S. Templeton. Phys. Rev. Lett. 1982. V. 48. P. 372.

[15] S. Deser, J. H. Kay. Phys. Lett. B. 1983. V. 120. P. 97.

[16] S. Deser. Phys. Lett. B. 1984. V. 140. P. 321.

[17] C. Aragone, A. Khoudeir. Phys. Lett. B. 1986. V. 173. P. 141.

[18] C. Aragone, J. Stephany. Class. Quantum. Gravit. 1984. V. 1. P. 265.

[19] C. Aragone, S. Deser. Class. Quantum. Gravit. 1984. V. 1. P. 331.

[20] M. А. Васильев. ЯФ. 1987. Т. 45. С. 1784; M. A. Vasiliev. Fortschr. Phys. 1988. V. 35. P. 741.

[21] Д. М. Гитман, И. В. Тютин. Каноническое квантование полей со связями. М.: Наука, 1986.

[22] M. A. Vasiliev. Phys. Lett. B. 1992. V. 285. P. 225.

[23] Ch. Devchand, V. Ogievetsky. Nucl. Phys. B. 1996. V. 367. P. 140.

Поступила в редакцию 7.IV.1997 г.

\section{A. Vasiliev, I. V. Tyutin \\ LAGRANGIAN FORMULATION OF IRREDUCIBLE MASSIVE FIELDS OF ARBITRARY SPIN IN $(2+1)$-DIMENSIONS}

Lagrangian formulation of the free massive fields corresponding to irreducible representations of the Poincaré group of arbitrary integer and half-integer spins in three-dimensional space-time is presented. A relationship of the theory under consideration with the self-duality equations in four dimensions is discussed. 